\title{
Edge Theorem for Multivariable Systems ${ }^{1}$
}

\author{
Long Wang ${ }^{1} \quad$ Zhizhen Wang ${ }^{1} \quad$ Lin Zhang ${ }^{2} \quad$ Wensheng Yu $^{3}$ \\ ${ }^{1}$ Center for Systems and Control, Department of Mechanics, Peking University, Beijing 100871, China \\ 2 Computer Science Department, Naval Postgraduate School, Monterey, CA93943, USA \\ ${ }^{3}$ Institute of Automation, Chinese Academy of Sciences, Beijing 100080, China
}

\begin{abstract}
This paper studies robustness of multivariable systems with parametric uncertainties, and establishes a multivariable version of Edge Theorem. An illustrative example is presented.
\end{abstract}

Keywords: Complex Systems, Robustness, Uncertain Parameters, Edge Theorem, Polynomial Matrices, Interval Polynomials.

\section{Introduction}

Motivated by the seminal theorem of Kharitonov on robust stability of interval polynomials $[1$, 2], a number of papers on robustness analysis of uncertain systems have been published in the past few years $[3,4,5,6,7,8,9,10]$. Kharitonov's theorem states that the Hurwitz stability of the real (or complex) interval polynomial family can be guaranteed by the Hurwitz stability of four (or eight) prescribed critical vertex polynomials in this family. This result is significant since it reduces checking stability of infinitely many polynomials to checking stability of finitely many polynomials, and the number of critical vertex polynomials need to be checked is independent of the order of the polynomial family. An important extension of Kharitonov's theorem is the edge theorem discovered by Bartlett, Hollot and Huang[4]. The edge theorem states that the stability of a polytope of polynomials can be guaranteed by the stability of its one-dimensional exposed edge polynomials. The significance of the edge theorem is that it allows some (affine) dependency among polynomial coefficients, and applies to more general stability regions, e.g., unit circle, left sector, shifted half plane, hyperbola region, etc. When the dependency among polynomial coefficients is nonlinear, however, Ackermann shows that checking a subset of a polynomial family generally can not guarantee the stability of the entire family[11, 12$]$.

For Hurwitz stability of interval matrices, Bialas 'proved' that in order to guarantee robust stability, it suffices to check all vertex matrices[13]. Later, it was shown by Barmish that Bialas' result was incorrect[14]. Kokame and Mori eastblished a Kharitonov-like result on robust Hurwitz stability of interval polynomial matrices[15], and Kamal and Dahleh established some robust stability criteria for MIMO systems with fixed controllers and uncertain plants[16].

In this paper, we will study robustness of a class of MIMO systems with their transfer function matrices described by

$$
\begin{aligned}
& \mathcal{F}(s)=\left\{\left(\begin{array}{lll}
a_{11}(s) & \ldots & a_{1 n}(s) \\
\ldots & \ldots & \ldots \\
a_{n 1}(s) & \ldots & a_{n n}(s)
\end{array}\right): a_{i j}(s) \in \mathcal{A}_{i j}(s)\right\} \\
& \mathcal{A}_{i j}(s)=\operatorname{conv}\left\{b 1_{i j}(s), \ldots, b m_{i j}(s)\right\}
\end{aligned}
$$

where $m$ is a given positive integer.

\section{Preliminaries}

Definition 1 A polynomial matrix is a matrix with all its entries being polynomials.

Definition 2 Suppose $D$ is a simply-connected region in the complex plane. If all the roots of the determinant of a polynomial matrix lie within $D$, then this polynomial matrix is called $D$-stable. A set of polynomial matrices is called robustly $D$-stable, if every polynomial matrix in this set is $D$-stable.

\footnotetext{
${ }^{1}$ Supported by National Natural Science Foundation of China(69925307). Email: longwang@mech.pku.edu.cn
} 
Definition 3 Suppose $f_{1}(s), \ldots, f_{m}(s)$ are $m$ given polynomials, the set

$$
\left\{\sum_{i=1}^{m} \lambda_{i} f_{i}(s): \quad \lambda_{i} \geq 0, \quad \sum_{i=1}^{m} \lambda_{i}=1\right\}
$$

is called the polynomial polytope generated by $f_{1}(s), \ldots, f_{m}(s)$, denoted as conv $\left\{f_{1}(s), \ldots, f_{m}(s)\right\}$.

Definition 4 The polynomial $f(s)=a_{0}+a_{1} s+\ldots+a_{n} s^{n}$, with $a_{i} \in\left[a_{i}^{L}, a_{i}^{U}\right]$ is called an interval polynomial.

Definition $5 S_{n}$ is the set of all bijections from $\{1, \ldots, n\}$ to $\{1, \ldots, n\}$.

Definition 6 The vertex set and edge set of $\mathcal{A}_{i j}(s)$ are

$$
\begin{gathered}
K_{i j}(s)=\left\{b 1_{i j}(s), \ldots, b m_{i j}(s)\right\} \\
E_{i j}(s)=\left\{\lambda b r_{i j}(s)+(1-\lambda) b t_{i j}(s), \quad \lambda \in[0,1], \quad r, t \in\{1, \ldots, m\}\right\}
\end{gathered}
$$

respectively.

\section{Definition 7}

$$
\mathcal{F}_{E}(s)=\bigcup_{\sigma \in S_{n}}\left\{\left(a_{i j}(s)\right)_{n \times n}: a_{i j}(s)\left\{\begin{array}{l}
\in E_{i j}(s) \text { if } i=\sigma(j) \\
\in K_{i j}(s) \text { if } i \neq \sigma(j)
\end{array}\right\}\right.
$$

Lemma 1 (Edge Theorem[4]) Suppose $\Gamma \subset \mathcal{C}$ is a simply-connected region, $\Omega$ is a polynomial polytope without degree dropping. Then, $\Omega$ is $\Gamma$-stable if and only if all the edges of $\Omega$ are $\Gamma$-stable.

Lemma 2 Suppose $A(s)$ is a given $n \times(n-1)$ polynomial matrix. Then

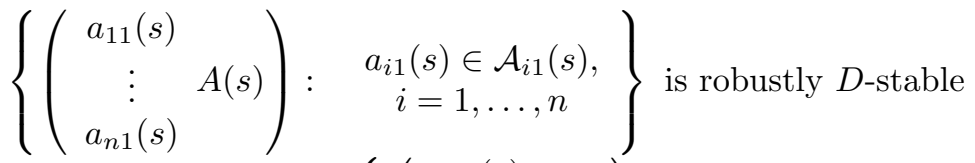

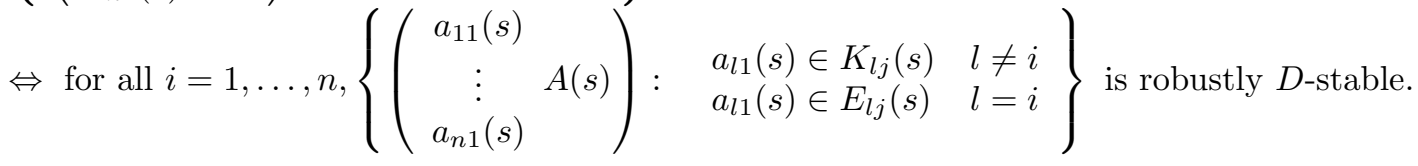

Proof: Necessity is obvious, since the later is a subset of the former.

Sufficiency: For any $a_{i 1}(s) \in \mathcal{A}_{i 1}(s)$, the corresponding matrix is

$$
T(s)=\left(\begin{array}{cc}
a_{11}(s) & \\
\vdots & A(s) \\
a_{n 1}(s) &
\end{array}\right)
$$

By Laplace Formula, we can expand the determinant of $T(s)$ along its first column. Then, by convexity and by Lemma 1 , we know that $T(s)$ is robustly $D$-stable.

Lemma 3 Suppose $B(s)$ is a given $(n-1) \times n$ polynomial matrix. $*$ stands for fixed entries in a matrix. Then

$$
\begin{aligned}
& \left\{\left(\begin{array}{ccc}
* & a_{1 i}(s) \underset{1 j}{*}(s) & * \\
& B(s)
\end{array}\right): \begin{array}{c}
a_{1 i}(s) \in \mathcal{A}_{1 i}(s) \\
a_{1 j}(s) \in \mathcal{A}_{1 j}(s)
\end{array}\right\} \text { is robustly } D \text {-stable }
\end{aligned}
$$

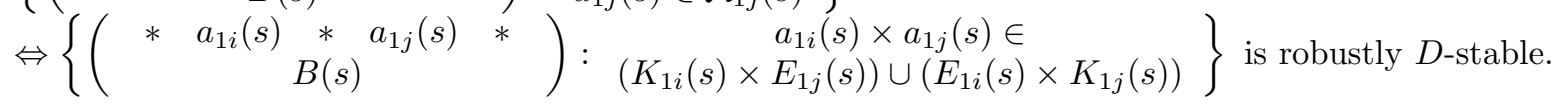

Proof: the proof is analogous to the proof of Lemma 2, except that the Laplace expansion is carried out along the row instead of the column. 


\section{$3 \quad$ Main Results}

Theorem $1 \mathcal{F}(s)$ is robustly $D$-stable if and only if $\mathcal{F}_{E}(s)$ is robustly $D$-stable.

Proof: Necessity is obvious. To prove sufficiency, we first note that interchanging any two rows (or columns) does not affect the stability of a polynomial matrix (it only changes the sign of the determinant). By Lemma 2

$$
\begin{aligned}
& \Leftrightarrow \text { for all } i=1, \ldots, n,\left\{\left(\begin{array}{cc}
a_{11}(s) \\
\vdots & \mathcal{F}(s) \text { is robustly } D \text { stable } \\
a_{n 1}(s)
\end{array}\right): \begin{array}{rl}
a_{l 1}(s) \in K_{l j}(s) & l \neq i \\
a_{l 1}(s) \in E_{l j}(s) & l=i
\end{array}\right\} \text { is robustly } D \text { stable. }
\end{aligned}
$$

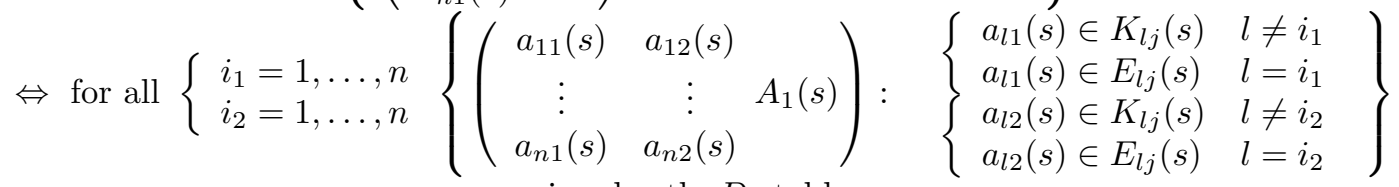

where $A_{1}(s)$ is the corresponding $n \times(n-2)$ polynomial matrix. This last equivalence is based on Lemma 2 and the fact that interchanging two columns does not change the stability of a polynomial matrix. Repeating the process above, let $Y_{n}$ denote the set of all mappings from $\{1, \ldots, n\}$ to $\{1, \ldots, n\}$, then

$$
\begin{aligned}
& \mathcal{F}(s) \text { is robustly } D \text { stable } \\
& \Leftrightarrow \text { for all } \eta \in Y_{n},\left\{\left(a_{i j}(s)\right): \begin{array}{ll}
a_{i j}(s) \in K_{i j}(s) & i \neq \eta(j) \\
a_{i j}(s) \in E_{i j}(s) & i=\eta(j)
\end{array}\right\} \text { is robustly } D \text { stable. }
\end{aligned}
$$

If there exists an $\eta \in Y_{n}$ such that $\eta\left(i_{1}\right)=\eta\left(i_{2}\right)=k$, then the corresponding matrix $F(s)=\left(a_{i j}(s)\right)$ satisfies

$$
\begin{aligned}
& a_{i_{1} k}(s) \in E_{i_{1} k}(s) \\
& a_{i_{2} k}(s) \in E_{i_{2} k}(s)
\end{aligned}
$$

Applying Lemma 2 to column $k$ of $F(s)$, we have

$\mathcal{F}(s)$ is robustly $D$ stable

$$
\begin{aligned}
& \Leftrightarrow \text { for all } \sigma \in S_{n},\left\{\left(a_{i j}(s)\right): \begin{array}{ll}
a_{i j}(s) \in K_{i j}(s) & i \neq \sigma(j) \\
a_{i j}(s) \in E_{i j}(s) & i=\sigma(j)
\end{array}\right\} \text { is robustly } D \text { stable. } \\
& \Leftrightarrow \mathcal{F}_{E}(s) \text { is robustly } D \text { stable. }
\end{aligned}
$$

\section{Interval Model}

Interval model, as a simple and effective approximation of uncertain systems, has been the subject of study in robustness analysis for a long time. In a similar vein, we consider the Hurwitz stability of the following uncertain system.

$$
\begin{aligned}
& \mathcal{G}(s)=\left\{\left(c_{i j}(s)\right): c_{i j}(s) \in \mathcal{C}_{i j}(s)\right\} \\
& \mathcal{C}_{i j}(s) \text { are interval polynomials }
\end{aligned}
$$

Definition 8 For the interval polynomial $\mathcal{C}_{i j}(s)=\left\{\sum_{l=0}^{n} q_{l}(i j) s^{l}, \quad q_{l}(i j) \in\left[\underline{q}_{l}(i j), \bar{q}_{l}(i j)\right]\right\}$, its Kharitonov vertex set and Kharitonov edge set are defined respectively as

$$
\begin{gathered}
K_{i j}^{I}(s)=\left\{c_{k}^{1}(s), c_{k}^{2}(s), c_{k}^{3}(s), c_{k}^{4}(s)\right\} \\
E_{i j}^{I}(s)=\left\{\lambda c_{k}^{r}(s)+(1-\lambda) c_{k}^{t}(s), \quad(r, t) \in\{(1,2),(2,4),(4,3),(3,1)\}, \quad \lambda \in[0,1]\right\}
\end{gathered}
$$

where

$$
\begin{array}{ll}
c_{k}^{1}(s)=\underline{q}_{0}(i j)+\underline{q}_{1}(i j) s+\bar{q}_{2}(i j) s^{2}+\bar{q}_{3}(i j) s^{3}+\ldots & c_{k}^{2}(s)=\underline{q}_{0}(i j)+\bar{q}_{1}(i j) s+\bar{q}_{2}(i j) s^{2}+\underline{q}_{3}(i j) s^{3}+\ldots \\
c_{k}^{3}(s)=\bar{q}_{0}(i j)+\underline{q}_{1}(i j) s+\underline{q}_{2}(i j) s^{2}+\bar{q}_{3} s^{3}+\ldots & c_{k}^{4}(s)=\bar{q}_{0}(i j)+\bar{q}_{1}(i j) s+\underline{q}_{2}(i j) v s^{2}+\underline{q}_{3}(i j) s^{3}+\ldots
\end{array}
$$




\section{Definition 9}

$$
\mathcal{G}_{E}(s)=\bigcup_{\sigma \in S_{n}}\left\{\left(c_{i j}(s)\right)_{n \times n}: c_{i j}(s)\left\{\begin{array}{l}
\in E_{i j}^{I}(s) \text { if } i=\sigma(j) \\
\in K_{i j}^{I}(s) \text { if } i \neq \sigma(j)
\end{array}\right\}\right.
$$

Lemma 4 (Box Theorem[16]) Suppose $\Delta(s)=\left\{\delta(s, p)=F_{1}(s) P_{1}(s)+\ldots+F_{m}(s) P_{m}(s)\right\}, P_{i}(s)$ is an interval polynomial, $F_{i}(s)$ is a given fixed polynomial, $i=1, \ldots, m$. And suppose $\Delta(s)$ is degree-invariant. Then, $\Delta(s)$ is Hurwitz stable if and only if $\Delta_{E}(s)$ is Hurwitz stable, where $\Delta_{E}(s)=$ $\cup_{l=1}^{m}\left\{\sum_{i=1}^{l-1} F_{i}(s) K_{P_{i}}^{0}(s)+F_{l}(s) E_{P_{l}}^{0}(s)+\sum_{i=l+1}^{m} F_{i}(s) K_{P_{i}}^{0}(s)\right\}\left(\right.$ let $\sum_{i=r}^{t} f_{i}=0$, if $\left.r>t\right)$.

By resort to the Box Theorem, and following a similar line of arguments as in the proof of Theorem 1 , we can get some analogous stability verification results for the interval model.

Lemma 5 Suppose $A(s)$ is a given $n \times(n-1)$ polynomial matrix. Then

$$
\begin{aligned}
& \left\{\left(\begin{array}{cc}
c_{11}(s) \\
\vdots & A(s) \\
c_{n 1}(s) &
\end{array}\right): \begin{array}{c}
c_{i 1}(s) \in \mathcal{G}_{i 1}(s), \\
i=1, \ldots, n
\end{array}\right\} \text { is robustly Hurwitz stable. }
\end{aligned}
$$

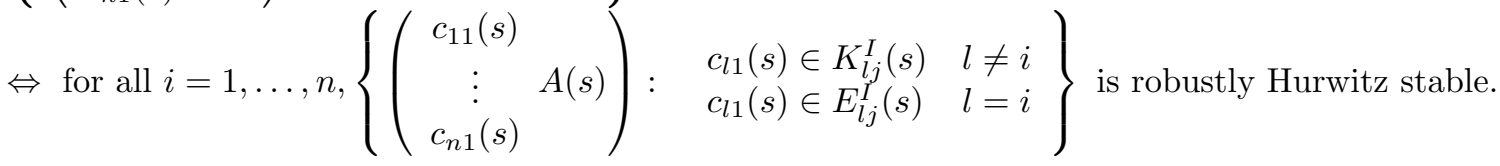

Lemma 6 Suppose $B(s)$ is a given $(n-1) \times n$ polynomial matrix. $*$ stands for fixed entries in a matrix. Then

$$
\begin{aligned}
& \left\{\left(\begin{array}{ccc}
* & c_{1 i}(s) \quad * \quad c_{1 j}(s) * \\
& B(s)
\end{array}\right): \begin{array}{l}
c_{1 i}(s) \in \mathcal{C}_{1 i}(s) \\
c_{1 j}(s) \in \mathcal{C}_{1 j}(s)
\end{array}\right\} \text { is robustly Hurwitz stable }
\end{aligned}
$$

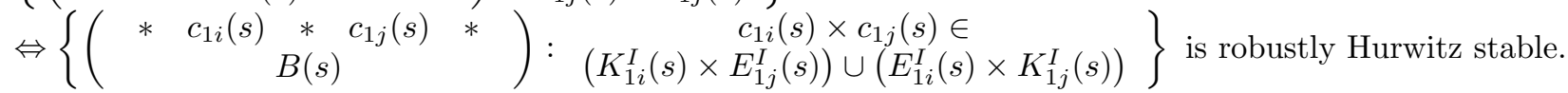

Theorem $2 \mathcal{G}(s)$ is robustly Hurwitz stable if and only if $\mathcal{G}_{E}(s)$ is robustly Hurwitz stable.

Remark: Theorem 2 is consistent with the result in [15]. In [15], the authors obtained their result using some theorem in signal processing. Our proof is based on the properties of matrix determinant, hence is more straightforward and self-contained.

\section{Example}

Consider the uncertain polynomial matrix

$$
\begin{aligned}
& \mathcal{A}(s)=\left\{\left(a_{i j}(s)\right)_{3 \times 3}\right\} \\
& a_{11}(s)=\lambda_{11} b 1_{11}(s)+\left(1-\lambda_{11}\right) b 2_{11}(s) \\
& a_{12}(s)=\lambda_{12} b 1_{12}(s)+\left(1-\lambda_{12}\right) b 2_{12}(s) \\
& a_{13}(s)=\lambda_{13} b 1_{13}(s)+\left(1-\lambda_{13}\right) b 2_{13}(s) \\
& a_{21}(s)=\lambda_{21} b 1_{21}(s)+\left(1-\lambda_{21}\right) b 2_{21}(s) \\
& a_{22}(s)=\lambda_{22} b 1_{22}(s)+\left(1-\lambda_{22}\right) b 2_{22}(s) \\
& a_{23}(s)=\lambda_{23} b 1_{23}(s)+\left(1-\lambda_{23}\right) b 2_{23}(s) \\
& a_{31}(s)=\lambda_{31} b 1_{31}(s)+\left(1-\lambda_{31}\right) b 2_{31}(s) \\
& a_{32}(s)=\lambda_{32} b 1_{32}(s)+\left(1-\lambda_{32}\right) b 2_{32}(s) \\
& a_{33}(s)=\lambda_{33} b 1_{33}(s)+\left(1-\lambda_{33}\right) b 2_{33}(s)
\end{aligned}
$$


Then,

$$
\begin{aligned}
& E_{i j}(s)=\left\{a_{i j}(s)\right\} \\
& K_{i j}(s)=\left\{b 1_{i j}(s), b 2_{i j}(s)\right\} \\
& S_{3}=\left\{\sigma_{1}, \ldots, \sigma_{6}\right\} \\
& \sigma_{1}: 1 \rightarrow 1 ; 2 \rightarrow 2 ; 3 \rightarrow 3 \\
& \sigma_{2}: 1 \rightarrow 2 ; 2 \rightarrow 3 ; 3 \rightarrow 1 \\
& \sigma_{3}: 1 \rightarrow 3 ; 2 \rightarrow 1 ; 3 \rightarrow 2 \\
& \sigma_{4}: 1 \rightarrow 1 ; 2 \rightarrow 3 ; 3 \rightarrow 2 \\
& \sigma_{5}: 1 \rightarrow 2 ; 2 \rightarrow 1 ; 3 \rightarrow 3 \\
& \sigma_{6}: 1 \rightarrow 3 ; 2 \rightarrow 2 ; 3 \rightarrow 1
\end{aligned}
$$

Let

$$
\mathcal{A}_{E}(s)=\bigcup_{\sigma \in S_{3}}\left\{\left(a_{i j}(s)\right)_{3 \times 3}: a_{i j}(s)\left\{\begin{array}{l}
\in E_{i j}(s) \text { if } i=\sigma(j) \\
\in K_{i j}(s) \text { if } i \neq \sigma(j)
\end{array}\right\}\right.
$$

By Theorem $1, \mathcal{A}(s)$ is robustly $D$ stable if and only if $\mathcal{A}_{E}(s)$ is robustly $D$ stable.

\section{Conclusions}

This paper discussed the robust $D$-stability problems for MIMO uncertain systems. The Edge Theorem and Kharitonov Theorem have been generalized to multivariable case.

\section{References}

[1] V.L.Kharitonov. Asymptotic stability of an equilibrium position of a family of systems of linear differential equations, Differential'nye Uravneniya, vol.14, 2086-2088, 1978.

[2] V.L.Kharitonov. The Routh-Hurwitz problem for families of polynomials and quasipolynomials, Izvetiy Akademii Nauk Kazakhskoi SSR, Seria fizikomatematicheskaia, vol.26, 69-79, 1979.

[3] C.V.Hollot and R.Tempo. On the Nyquist envelope of an interval plant family, IEEE Trans. on Automatic Control, vol.39, 391-396, 1994.

[4] A.C.Bartlett, C.V.Hollot and L.Huang. Root locations of an entire polytope of polynomials: It suffices to check the edges, Mathematics of Control, Signals, and Systems, vol.1, 61-71, 1988.

[5] M.Fu and B.R.Barmish. Polytope of polynomials with zeros in a prescribed set, IEEE Trans. on Automatic Control, vol.34, 544-546, 1989.

[6] L. Wang and L. Huang. Vertex results for uncertain systems, Int. J. Systems Science, vol.25, 541-549, 1994.

[7] L. Wang and L. Huang. Extreme point results for strict positive realness of transfer function families, Systems Science and Mathematical Sciences, vol.7, 371-378, 1994.

[8] B. R. Barmish, C. V. Hollot, F. J. Kraus and R. Tempo. Extreme point results for robust stabilization of interval plants with first order compensators, IEEE Trans. on Automatic Control, vol.37, 707-714, 1992.

[9] H. Chapellat, M. Dahleh and S. P. Bhattacharyya. On robust nonlinear stability of interval control systems, IEEE Trans. on Automatic Control, vol.36, 59-67, 1991.

[10] L. Wang and L. Huang. Finite verification of strict positive realness of interval rational functions, Chinese Science Bulletin, vol.36, 262-264, 1991.

[11] J.Ackermann. Uncertainty structures and robust stability analysis, Proc. of European Control Conference, 2318-2327,1991. 
[12] J.Ackermann. Does it suffice to check a subset of multilinear parameters in robustness analysis? IEEE Trans. on Automatic Control, vol.37, 487-488, 1992.

[13] S. Bialas. A necessary and sufficient condition for the stability of interval matrices, Int. J. Control, vol.37, 717-722, 1983.

[14] B. R. Barmish and C. V. Hollot. Counter-example to a recent result on the stability of interval matrices by S. Bialas, Int. J. Control, vol.39, 1103-1104, 1984.

[15] H. Kokame and T. Mori. A Kharitonov-like theorem for interval polynomial matrices, Systems and Control Letters, vol.16, 107-116, 1991.

[16] F. Kamal and M. Dahleh. Robust stability of multivariable interval control systems, Int. J. Control, vol.64, 807-828, 1996. 